\title{
Laser Frenectomy of Papilla Penetrating Frenum - A Case Report
}

\section{Shruti Pandey*}

Dental Surgeon, Dr. Shruti's Dental Care, Gujarat, India

*Corresponding Author: Shruti Pandey, Dental Surgeon, Dr. Shruti's Dental Care, Gujarat, India.

Received: September 19, 2019; Published: October 11, 2019

DOI: $10.31080 /$ ASDS.2019.03.0668

\begin{abstract}
Aberrant labial frenum attachment hampers function and aesthetics in maxillary anterior region which can be managed by frenectomy or frenotomy using conventional scalpel incisions or Laser. We preferred to use diode laser due to its extremely precise cutting, clear and bloodless operating field, fast ablation, instant hemostasis, predictable tissue response and less postoperative pain due to coagulation effect on small blood vessels and sensory nerve endings reducing the use of analgesics and pre-operative anaesthesia.

We present a case of midline diastema caused by papilla penetrating labial frenulum attachment treated with diode laser frenectomy.

Keywords: Laser; Labial Frenum; Anaesthesia
\end{abstract}

\section{Introduction on Labial Frenum and Frenectomy}

Frenum is a fan shaped fold of mucous membrane, usually with enclosed muscle fibres that attaches the lips and cheeks to the alveolar mucosa and/or gingiva or periosteum. Labial frenum is classified on basis of the extension of attachment of frenal fibres, i.e., Mucosal, Gingival, Papillary and Papilla penetrating by Placek., et al.

- Mucosal: Frenal fibres attached upto mucogingival junction

- Gingival: Fibres inserted with attached gingiva

- Papillary: Fibres extending into mucogingival junction

- Papilla penetrating: When frenal fibres cross the alveolar process and extend upto palatine/incisive papilla.

\section{Blanch test}

The attachment can be confirmed by performing blanch test, using index fingers and thumbs lift the upper lip and extend the frenum and look for blanching near the frenal attachment and attached gingiva. If extensive blanching is present, frenal attachment is wide and pathogenic.

If there is no apparent zone of attached gingiva along the midline or if the interdental papilla shifts upon extending the labial frenum, the frenum is considered pathogenic according to Miller.

Such pathogenic frenum causes gingival recession followed by midline diastema, difficulty in retaining maxillary denture.

We present a case of midline diastema caused by papilla penetrating labial frenulum attachment treated with diode laser frenectomy in this article.

\section{Case Report}

A 26-years-old male Indian doctor reported with a chief complain of space/gap between upper anterior/front teeth i.e., maxillary central incisors. He preferred orthodontic treatment over various options.

On intraoral examination, midline diastema of $4 \mathrm{~mm}$ spacing and papilla penetrating high frenal fan shaped and dumbell shaped attachment were observed. Patient was advised for frenectomy followed by clear aligners.

Laser Frenectomy procedure was explained in detail and written consent was obtained.

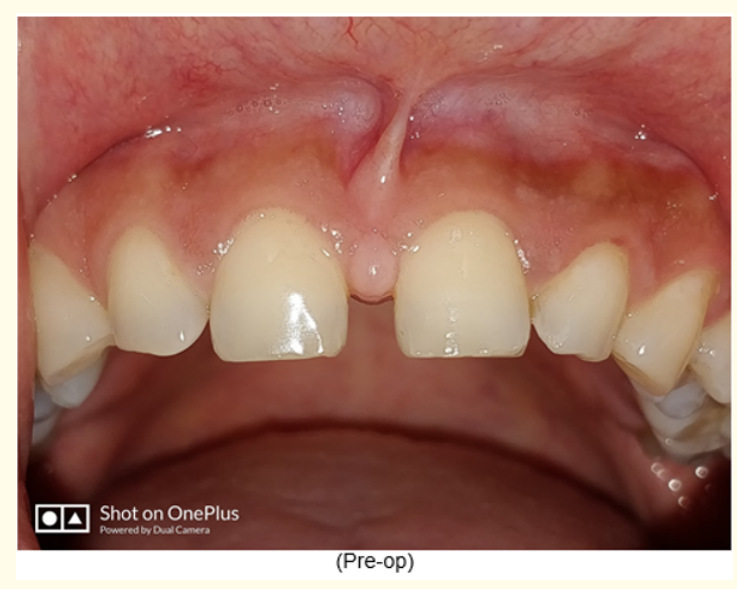

Figure 1: Fan shaped Papilla penetrating type of frenal attachment before frenectomy; labial view. 


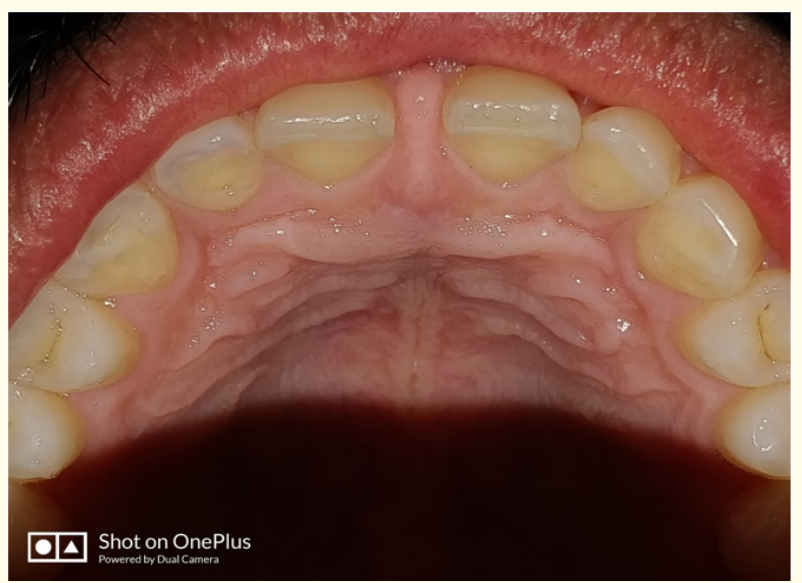

Figure 2: Dumbell shaped papilla penetrating type of frenal attachment before frenectomy; occlusal.

Procedure and laser parameters

Labial frenectomy was performed using biolase diode laser with a wavelength of $980 \mathrm{~nm}$, surgical tip $400 \mu \mathrm{m}$ with power of $1.37 \mathrm{~W}$ in contact mode.

Topical anaesthesia (lignocaine 2\%) was applied over the frenal attachment and $2 \mathrm{ml}$ of lignocaine hydrochloride with adrenaline IP as adrenaline bitartrate IP (LIGNOX 2\% A) was injected using local infilteration anaesthesia technique with luer lock sterilised syringe of 26 gauge needle, $1 \mathrm{ml}$ each labial and palatal surface.

Laser was activated before performing the procedure.

A rhomboid/football shaped incision was made from the frenum to attached gingiva and inner side of the lip, extending to the depth of the labial vestibule on labial surface and upto incisive papilla tip on occlusal surface due to papilla penetrating type of frenal attachment, causing separation of fibres and release of tension which was causing blanching. Blanch test was repeated and no blanching was seen.

Hemostasis was optimal. No sutures were given. Patient, doctor and assistant wore laser safety goggles.

\section{Post-operative instructions}

Patient was advised to avoid hot and spicy food for at least a week and maintain meticulous oral hygiene. Lukewarm saline gargles were advised thrice a day for 5 days after a week of procedure. Patient was advised to use chlorhexidine mouthwash thrice a day postoperatively for a week and avoid brushing in the operated area for three weeks. Analgesic ketorolac thromethamine $10 \mathrm{mg}$ BID for two days was prescribed, but patient reported with no post-operative pain and chose not to take any of them.

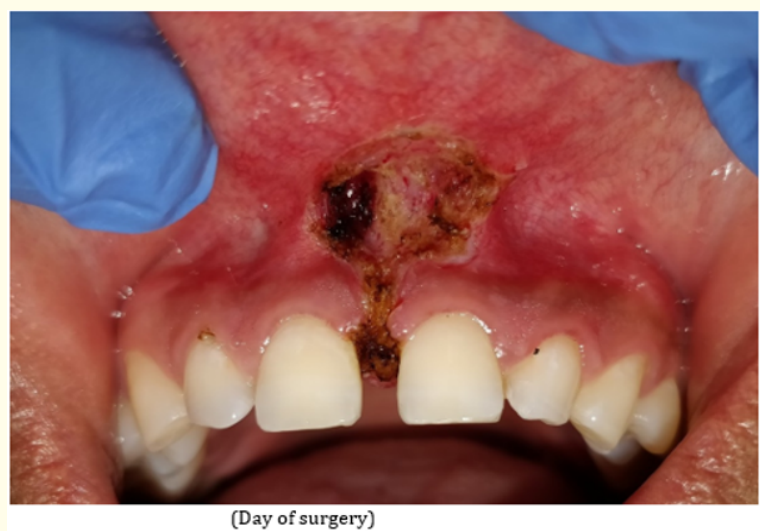

Figure 3: Diode LASER frenectomy; immediate post-operative image post-operative instructions.

Complete healing of the operated site was reported after three weeks.

Patient had no intraoperative complication such as pain, swelling and bleeding.

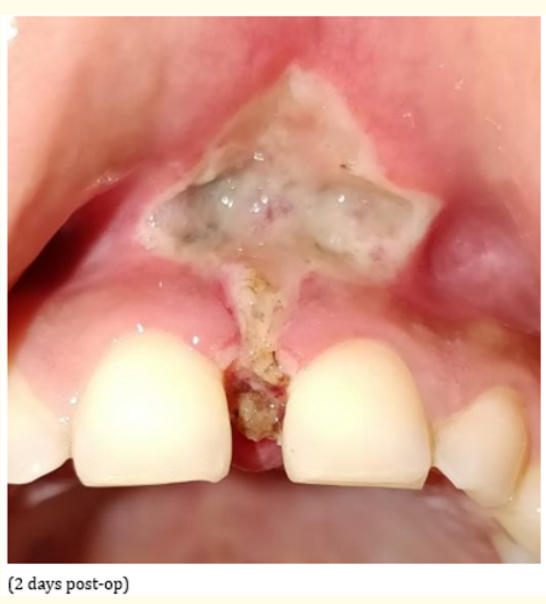

Figure 4: Two days Post-operative image of laser labial frenectomy.

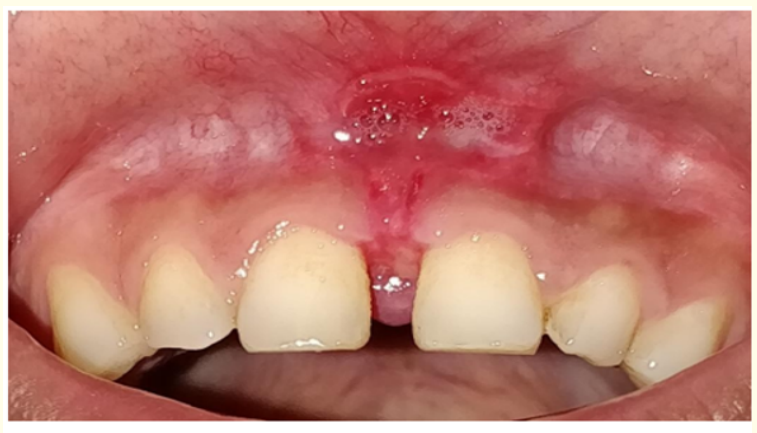

(3 weeks post-op)

Figure 5: Three weeks post-operative image of Laser labial frenectomy. 


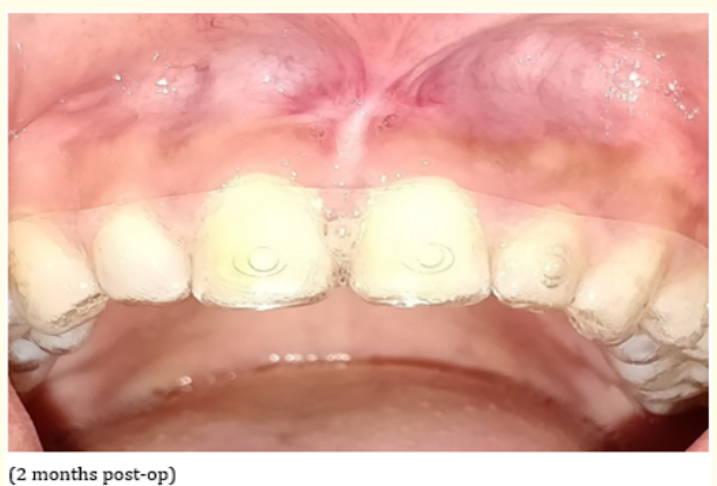

Figure 6: Two months post-operative new frenal attachment, Patient is wearing third set of his clear aligners for midline diastema closure (orthdontic treatment)/straightening of his teeth.

\section{Principle of lasers}

Diode lasers works on the principle of photothermal interaction which are composed of semiconductor crystals Iridium/ Aluminum, arsenic, gallium with a wavelength range of $810 \mathrm{~nm}$ to $1064 \mathrm{~nm}$. Their wavelength approximates with the absorption coefficient of melanin, hemoglobin, collagen and chromophores so they are best suited for soft tissue surgeries.

\section{Advantages of Laser frenectomy over conventional frenectomy}

Lasers over conventional scalpel has minimally invasive approach that provides rapid hemostasis of small blood vessels, seals the sensory nerve endings that results in reduced postoperative pain and inflammation and formation of protein layer to protect the surgical site from external stimuli reducing irritation. Bloodless field of surgery was observed due to its coagulation effect on small blood vessels on certain wavelength (here $980 \mathrm{~nm}$ ) and tissue contact omitting the use of sutures. It also has an advantage of less patient discomfort, reduced use of analgesics, physical and mental well being of the patient, reduced post-operative oedema, reduced patient appointments, pain control and minimal blood loss [1-4].

\section{Conclusions}

Fiberoptic delivery, ease of use, minimally invasive approach, bloddless field of surgery, small size, less armamentarium, less patient discomfort makes laser the best choice for frenectomy among surgeons over conventional scalpel in $21^{\text {st }}$ century.

\section{Bibliography}

1. Newman M., et al. "Periodontal plastic and esthetic surgery". In: Carranza FA, editor. Carranza's Clinical Periodontology. 10th ed. MO: Saunders: St. Louis, Missouri: An Imprint of Elsevier Science (2006): 1023-1024.
2. Coluzzi DJ. "Fundamentals of dental lasers: Science and instruments". Dental Clinics of North America 48 (2004): 751-770.

3. Placek M., et al. "Significance of the labial frenal attachment in periodontal disease in man. Part 1; Classification and epidemiology of the labial frenum attachment". Journal of Periodontology 45 (1974): 891-894.

4. Patil P., et al. "Diode laser frenectomy: A case report with review of literature". Journal of Dental Lasers 13 (2019): 19-22.

\section{Volume 3 Issue 11 November 2019 \\ (C) All rights are reserved by Shruti Pandey.}

(1) 